\title{
A Modern Standardized Method for Predicting Community Response to Aircraft Noise
}

\author{
Sanford Fidell \\ Fidell Associates, Inc., 23139 Erwin Street, Woodland Hills, United States
}

Copyright $(2018$ by authors, all rights reserved. Authors agree that this article remains permanently open access under the terms of the Creative Commons Attribution License 4.0 International License

\begin{abstract}
National (and in some cases, state) legislation in the United States requires prediction of community response to aircraft noise exposure as part of the disclosure of environmental effects of proposed construction of airport infrastructure. Formulation of transportation noise policy and systematic regulation of transportation noise require similar predictions. Historically, such predictions have been based on dosage-response functions derived from univariate correlational analyses in which cumulative noise exposure serves as the sole predictor of annoyance. Such functions typically ignore major differences in annoyance prevalence rates in communities with similar levels of noise exposure, and leave much of the variance in the relationship between exposure and community response unaccounted for. More complex regression models with additional predictor variables can account for more variance, but are ill-suited for regulatory purposes. A recently revised international standard, ISO 1996-1:2016, describes a causal (rather than correlational) prediction method known as Community Tolerance Level ("CTL") that addresses the limitations of dosage-response functions derived by univariate regression modeling. CTL-based predictions of the prevalence of a consequential degree of aircraft noise-induced annoyance can account for notably more variance in the relationship between noise exposure and annoyance than univariate regression predictions. The CTL approach also provides a consistent rationale for defining the significance of noise exposure, and a systematic approach to regulation of transportation noise.
\end{abstract}

Keywords Transportation Noise, Community Response to Noise, Noise-induced Annoyance, Noise Regulation, CTL

\section{Objective}

This tutorial describes the Community Tolerance Level (abbreviated CTL, and represented symbolically in mathematical expressions as $L_{\mathrm{ct}}$ ) approach to estimating the prevalence of transportation noise-induced annoyance in communities. It also distinguishes the approach, and the dosage-response functions that it yields, from earlier, regression-based curve fits. It further shows how CTL analysis permits systematically-derived regulatory policy.

\section{Introduction}

Airports are essential elements of air transportation networks that are frequently described by their proponents as engines of economic development. In economic terms, however, residential exposure to aircraft noise in airport neighborhoods is a "negative externality": a cost that is not fully reflected in passenger airfares, air freight charges, and airport operating budgets. Opportunity costs, such as restriction of real estate development and other costs associated with aviation-related land use zoning, in combination with losses of property tax revenue to local jurisdictions, are among the unintended consequences of airport operation that are rarely reflected in airport economic impact analyses. At smaller airports, these economic and political costs may be so extensive that they can challenge the assumption that aviation-related land uses are the best and highest for planning purposes.

Informed regulation of aircraft noise exposure in the vicinity of airports seeks to balance conflicting societal interests in support for air transportation services on the one hand, against interests in habitable and sustainable residential neighborhoods on the other hand. Such regulation requires an answer to the question "How much noise is too much noise?" A simplistic answer to the question is often expressed in the form of a single, fixed threshold of "significance" of cumulative, long-term aircraft noise exposure. The U.S. Federal Aviation Administration ("FAA") and other transportation noise regulatory agencies have adopted the position that the prevalence of a consequential degree of aircraft 
noise-induced annoyance in airport neighborhoods is the preferred measure of adverse impact of noise on communities [1]. FAA's primary criterion of significance of aircraft noise exposure is a level of Day-Night Average Sound Level (an A- and time-weighted 24 hour energy average measure) of $65 \mathrm{~dB}$.

Dosage-response functions have been considered since 1978 [2] as the most useful basis for prediction of the prevalence of a consequential degree of transportation noise-induced annoyance. Such functions are commonly derived by univariate logistic regression, and rest on the assumption that the prevalence of annoyance in communities may be usefully modeled as due exclusively to long-term, cumulative noise exposure, as quantified by noise metrics such as Day-Night Average Sound Level (abbreviated DNL, and represented symbolically in mathematical expressions as $L_{\mathrm{dn}}$.) FICON's [1] dosage-response function associates a prevalence rate of transportation noise-induced annoyance of $12.3 \%$ of the population with a DNL value of $65 \mathrm{~dB}$. In effect, FAA thus considers that cumulative, long-term aircraft noise exposure at a level that highly annoys $12.3 \%$ of the population constitutes a "significant" noise impact.

Annex $\mathrm{H}$ of a recently published international technical consensus standard [3] identifies a different dosage-response function for relating transportation noise exposure to the prevalence of a high degree of annoyance in communities. This function is not derived from field observations by correlation-based curve fitting methods. Instead, it is derived from a causal model which assumes that the prevalence of high annoyance in communities grows with noise exposure at a rate similar to that at which loudness grows with sound level.

The method described in Annex H of ISO 1996-1:2016 parses annoyance prevalence rates observed in communities into two components. One component is the effective (i.e., duration-adjusted) loudness of noise exposure. The other component is the aggregate influence of all non-acoustic factors on self-reports of annoyance. This paper reviews and explains the development and use of the methods described in Annex H of [3].

\section{Background}

Fidell [4] has traced formal efforts to quantify the effects of aircraft noise on communities to the introduction of jet propulsion to military aviation in the early 1950s. Rosenblith et al. [5] devised a decibel-like "Community Noise Rating" (CNR) scale of aircraft noise exposure, and identified a value of 100 on this scale as a point above which noise exposure was likely to trigger complaints and threats of litigation. The CNR scale was subsequently converted into a "Noise Exposure Forecast" (NEF) scale, on which a value of $30 \mathrm{~dB}$ was mathematically equivalent to a $\mathrm{CNR} \approx 100$ value. The CNR and NEF values that were identified as thresholds of significant noise effect were not derived from any systematic rationale, nor from extensive empirical evidence. Rather, they were simply the opinions of prominent acoustical engineers of the era about levels of noise exposure that would permit airport proprietors to avoid unmanageable numbers of complaints and threats of litigation.

About a quarter of a century after the Rosenblith et al. research of the early 1950s, Schultz [2] provided a rationale for converting the goal of aircraft noise regulation from management of complaints to protection of airport communities from exposure to highly annoying noise. Fidell [4] notes that "Schultz demonstrated that the results of social surveys conducted in disparate cities and languages on the effects of aircraft and surface transportation noise could be interpreted in common terms, and usefully summarized in the form of a dosage-effect relationship."

The U.S. FAA and the Federal Interagency Committee on Noise (FICON) formally endorsed Schultz's research by asserting that their definitions of "significant" noise exposure were based on an updated version of Schultz's analyses. In 1992, FICON [1] formally endorsed a particular dosage-response function derived by conventional curve-fitting means. Billions of dollars have since been spent in the United States on mitigation of "significant" aircraft noise impacts in neighborhoods near airports, largely through residential acoustic insulation programs and private property purchases.

Inconveniently, the findings of social surveys conducted since 1961 on the annoyance of aircraft noise annoyance have proven to be highly variable, and in poor agreement with the FICON curve. Figure 1 summarizes field observations of the prevalence of a consequential degree of self-reported annoyance with aircraft noise exposure in 80 social surveys. Each plotting symbol represents a paired, empirical observation of a community's noise exposure with the prevalence of high annoyance with aircraft noise in that community.

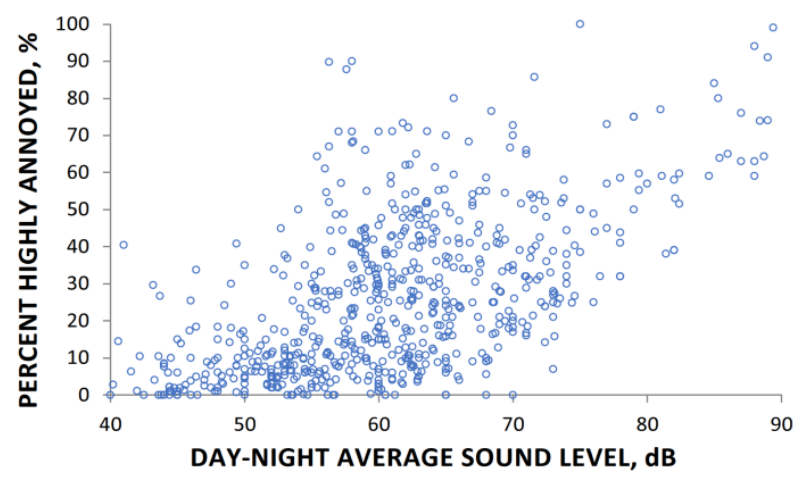

Figure 1. Empirical observations of the prevalence of aircraft noise-induced annoyance in communities world-wide.

The great variability of community response to aircraft noise from one community to the next that is apparent in 
Figure 1 is the most striking aspect of these data. ${ }^{1}$ No dosage-response relationship intended to summarize these data that is derived from correlation-based analysis, such as univariate regression, has succeeded in accounting for the better part of the variance in this data set.

A minor part of the variability apparent in Figure 1 could be due to various forms of error of measurement on both axes. Some of the variability may also stem from individual- (rather than community-) level factors. Transportation noise in communities propagates to all individuals' residences, however, and individual-level influences on annoyance prevalence rates are unknowable in advance for all practical purposes. Individual-level explanatory factors ("co-variates" in regression analysis) are thus of meager utility for predictive purposes. The great bulk of the variability in community response to transportation noise exposure most likely reflects bona fide, community-specific differences in reactions to aircraft noise exposure.

At best, a regression-derived dosage-response relationship can represent the central tendency of the relationship between aircraft noise exposure and annoyance in many communities. The variability in the underlying data is so great, however, that no single, "one-size-fits-all" dosage-response relationship can accurately predict the prevalence of annoyance with aircraft noise in any specific community.

Legislation such as the U.S. National Environmental Policy Act nonetheless requires that proponents of airport infrastructure construction projects disclose the specific impacts of proposed projects on specific neighborhoods, not the generic effects of aircraft noise on a hypothetically typical community. A generic statistical modeling tool, such as logistic regression, is less useful in such cases than a straightforward causal model that predicts how a community should respond to transportation noise exposure, and attributes deviations from expected behavior to the aggregate effect of factors other than exposure.

Annex H of ISO 1996-1 [3] describes an alternate approach (described below) to predicting the prevalence of transportation noise-induced annoyance that is based on such a causal model, rather than on generic curve-fitting methods.

\section{Method}

Appendix A of Fidell et al. [6] states the CTL approach in a simple set of equations. The basic predictive relationship is shown below:

$$
p(\mathrm{HA})=e^{(-A / m)}
$$

\footnotetext{
${ }^{1}$ It should also be noted that without field observations made at DNL values in excess of about $70 \mathrm{~dB}$, a strong correlation between noise exposure and the prevalence of high annoyance is not readily apparent.
}

where

$p(\mathrm{HA})$ is the proportion of the population highly annoyed by noise exposure, $A$ is a community's annoyance decision criterion ${ }^{2}$, and $m$ is the noise dose, defined as:

$$
\mathrm{m}=\left(10^{\left(\mathrm{L}_{\mathrm{dn}} / 10\right)}\right)^{0.3}
$$

Empirically, the proportion of a community highly annoyed is determined in field settings by direct questioning in a structured social survey interview. ${ }^{3}$ The functional relationship specified by the basic predictive relationship of Eq. [1] is a sigmoid. The exponential form of the relationship was chosen as the most plausible and parsimonious (i.e., single parameter) transition function to model the growth, from zero to one, of the proportion of a community highly annoyed by increasing levels of noise exposure.

CTL analysis treats the proportion of a community that describes itself as highly annoyed as equally influenced 1) by noise exposure per se, and 2) by a non-acoustic criterion for self-reporting of annoyance. Univariate regression analyses ignore the influence of the second of these determinants of annoyance prevalence rates. In CTL analysis, the slope of the dosage-response relationship is fixed at that of the exponential growth rate of loudness with sound level, while the parameter $A$ in Eq. [1] translates the sigmoidal function along the abscissa.

A family of CTL curves may be seen in Figure 2 over the range of DNL values that are of primary regulatory concern. All of the curves are of the form $e^{(-x)}$. They do not appear to be parallel within the plotted range of DNL values, because shifting them left and right along the abscissa displays different portions of the sigmoid in the plotting window.

In practice, the value of $A$ for a particular community is determined empirically, from social survey findings reported as the proportion of a community's residential population describing itself as highly annoyed by noise exposure for a given DNL. A Community Tolerance Level is defined as the value of DNL at which 50 percent of the population is expected to describe itself as highly annoyed by noise exposure. CTL values may be calculated from the value of A that produces the smallest RMS error ${ }^{4}$, as shown in Equation 3:

\footnotetext{
${ }^{2}$ The "annoyance decision criterion" is a scalar quantity that reflects the sum of all non-acoustic factors that influence decisions of survey respondents resident in the same communities to describe themselves as "highly annoyed" by noise exposure.

3 An ISO Technical Specification [7] offers procedural recommendations for the conduct of such surveys.

${ }^{4}$ Taraldsen et al. [10] have more recently suggested that a maximum likelihood ratio criterion is preferable to a minimal rms criterion for finding values of A. The method of Taraldsen et al. for estimating A is most appropriate for cases in which numbers of completed interviews vary greatly for different pairs of (DNL, \%HA) observations Differences in CTL between the two estimation methods are typically on the order of 1 or $2 \mathrm{~dB}$.
} 


$$
L_{c t}=33.3 \log _{10}(A)+5.32
$$

The panels of Figure 3 show sample fits of social survey data to the CTL function for six airports. Note that these fits are not derived by any correlation-based curve fitting method, but are simply those achieved by shifting an effective loudness function along the abscissa. Additional examples may be found in [8] and [9].

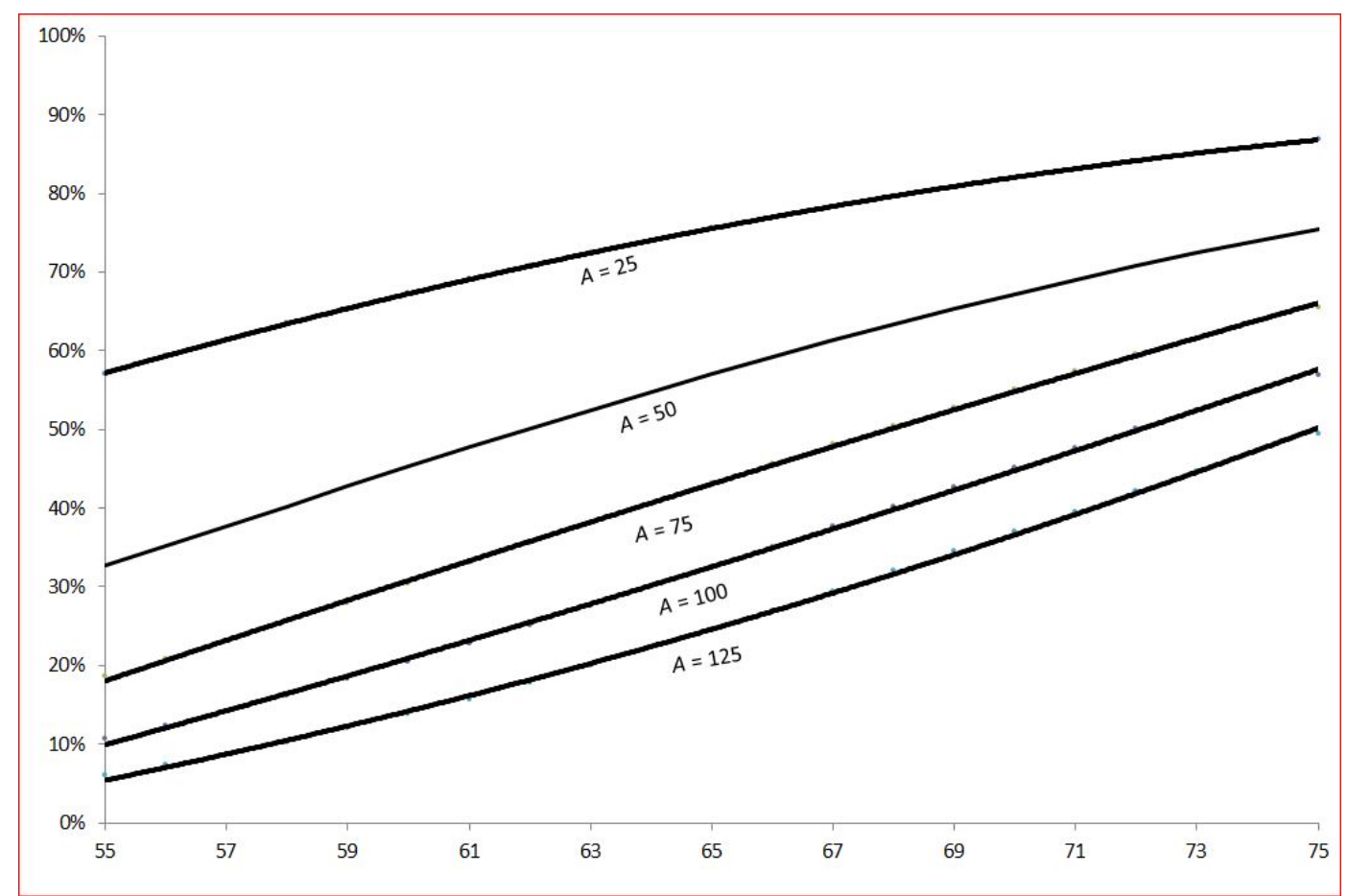

Figure 2. A family of dosage-response functions, parametric in $A$ (the non-acoustic response criterion parameter), relating cumulative 24 hour noise exposure to the prevalence of a consequential degree of transportation noise-induced annoyance in a community.
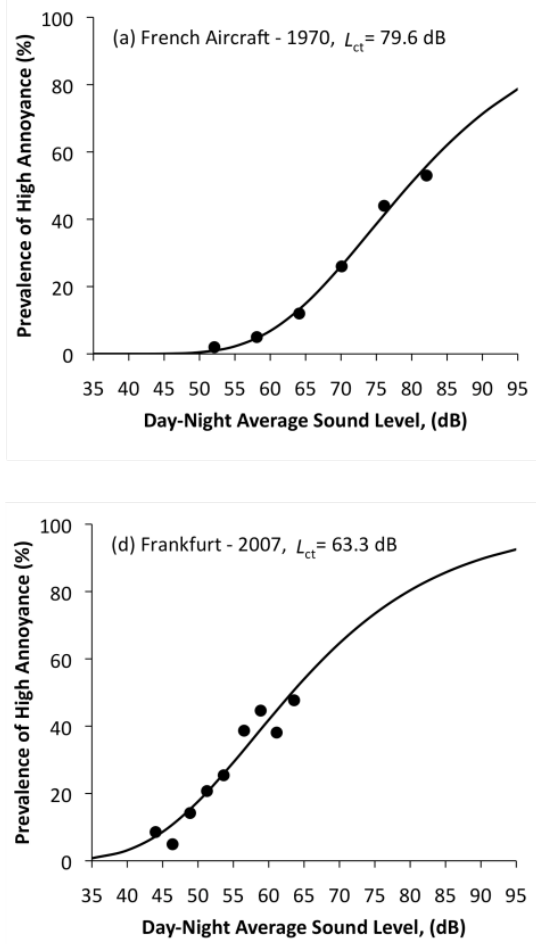
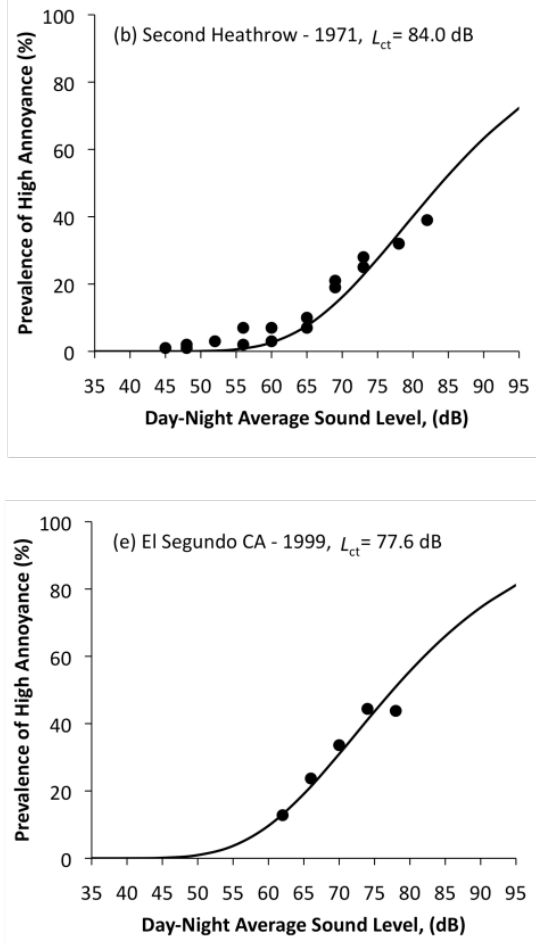
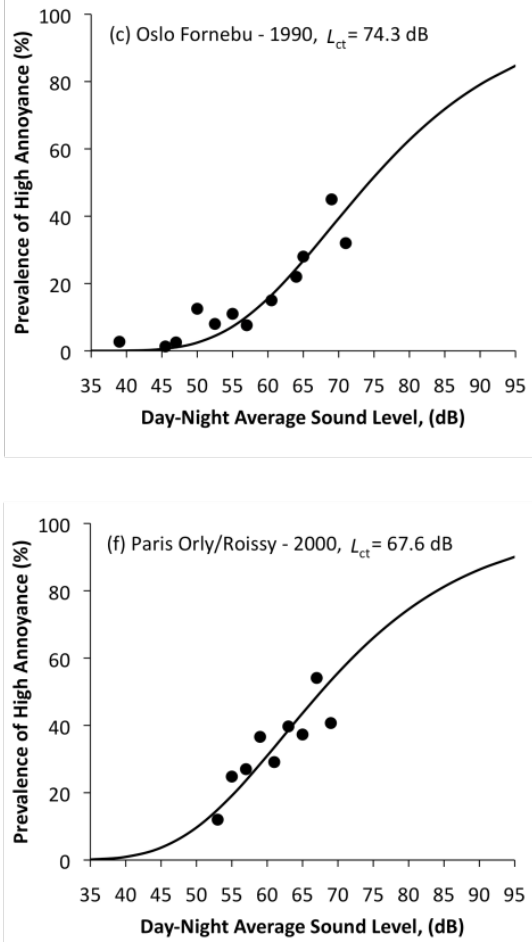

Figure 3. Examples of fit of field measurements of prevalence of high annoyance to effective loudness curve for six studies of community reaction to aircraft noise, from data compiled in [8]. 


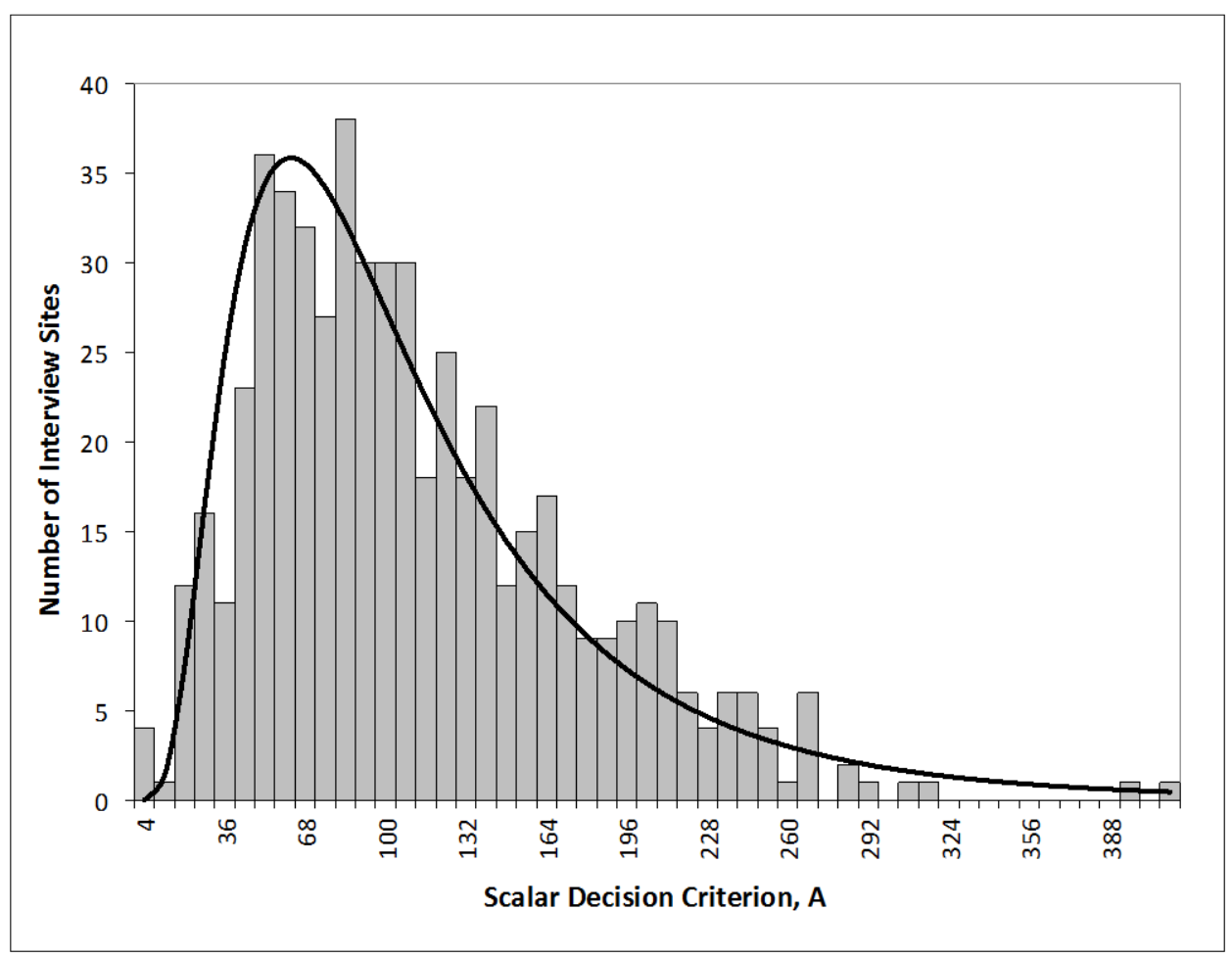

Figure 6. Distribution of the scalar quantity, $A$, over communities for annoyance due to aircraft noise

If CTL for a community is known, then the annoyance decision criterion, $A$, may be calculated from CTL as shown in Equation 4:

$$
\mathrm{A}=10^{\left(\frac{\mathrm{L}_{\mathrm{ct}}-5.32}{33.3}\right)}
$$

The distribution of $A$ for annoyance due to aircraft noise over communities is approximately log normal, as shown in Figure 6, adapted from [6]. Most communities show relatively little tolerance for exposure to aircraft noise, but a few are highly tolerant of it. ssCommunity tolerance for aircraft noise may reflect attitudes of misfeasance on the part of airport proprietors, fears of crashes, economic dependence on airport operation, or other individual-level concerns of airport neighborhood residents.

For broad regulatory purposes, however, the exact combination of reasons that particular communities are intolerant of aircraft noise is of secondary concern at best. Aircraft overfly residential areas of all types near airports, and over all residents of neighborhoods - men and women, young and old, sensitive and insensitive - regardless of their attitudes toward airport operation. Further, the proportions of community residents that are highly annoyed by aircraft noise for particular non-acoustic reasons at particular airports can never be known a priori.

For purposes of setting national transportation noise policy, the most practical and defensible regulatory strategy is therefore to separately identify the acoustically and non-acoustically influenced components of noise-induced annoyance, and to address the aggregate effects of all non-acoustic determinants of annoyance in the aggregate. Attempts to do otherwise - for example, to identify adjustments to national policies for airport neighborhoods with specified combinations of non-acoustic influences on annoyance - would lead to unworkably complex regulation. As noted below, CTL analysis additionally provides a statistical rationale for defining a "significant" amount of transportation noise exposure.

\section{Discussion}

The definition of a level of noise exposure that constitutes a "significant" amount of exposure is an expressly non-technical decision that must be based on political value judgments. As illustrated in Figure 1, however, a one-size-fits-all policy decision about "how much noise is too much noise" that works as intended in one particular community will not work well in many others. Although uniformity of regulation is a superficially attractive goal, uniformity of regulatory effect is a yet more substantive one. If protection of residential populations from exposure to highly annoying noise exposure is the major concern of aircraft noise regulatory policy, then a one-size-fits-all policy clearly cannot confer equal regulatory protection on all communities. Fidell et al. [6] demonstrate how "regulatory policy positions can be derived from two parameters: 1) the minimal percentage of the population of a nominally average community to be protected from exposure to highly annoying noise; and 2) the percentage of all communities to which this degree of protection is intended to apply." 


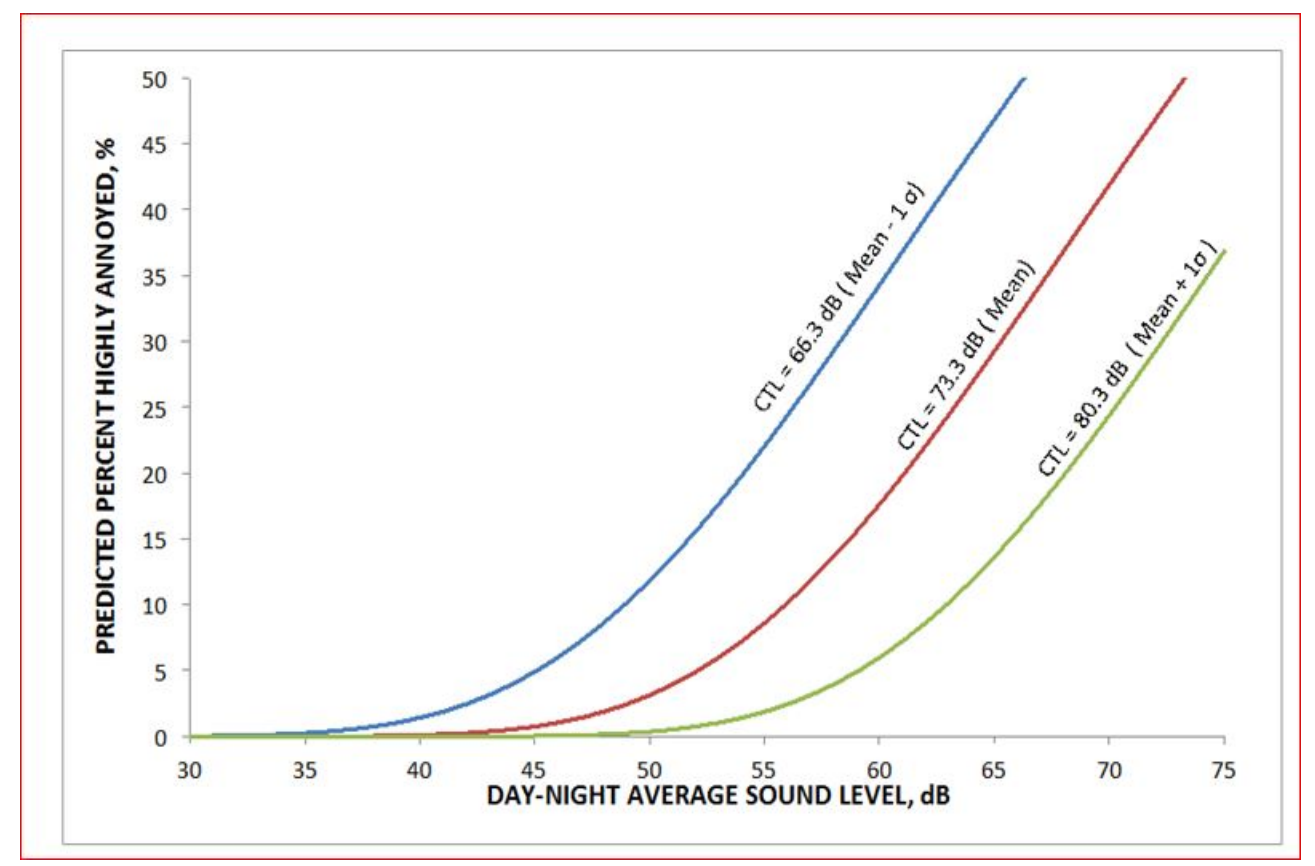

Figure 7. Dosage-response functions derived by CTL analysis for communities of average tolerance for aircraft noise exposure, and for tolerances \pm 1 standard deviation from the mean

The rationale for basing regulatory policy on CTL-based analysis requires knowledge of the mean and standard deviation of the distribution of CTL values over communities. For the studies included in the database published by Fidell et al. [8], the mean CTL value is about $73 \mathrm{~dB}$, and the standard deviation is about $7 \mathrm{~dB}$. The corresponding dosage-response functions relating to the prevalence of aircraft noise-induced high annoyance in a nominally average community, and in communities with CTL values $\pm 1 \sigma$ from the mean, are plotted in Figure 7 .

Table 1, adapted from [6], shows the percentages of residents of communities with average and $\pm 1 \sigma \mathrm{CTL}$ values who are protected from exposure to highly annoying noise by regulatory policies with various definitions of "significant" noise exposure. It is apparent that the current U.S. definition of the significance of aircraft noise exposure $\left(L_{\mathrm{dn}}=65 \mathrm{~dB}\right)$ leaves almost half of the residential populations of communities with average tolerance for aircraft noise unprotected from exposure to highly annoying noise.

Table 1. Percentages of population protected from exposure to highly annoying aircraft noise (100 - percent highly annoyed) for communities with average and CTL values \pm 1 standard deviation from the mean

\begin{tabular}{|c|c|c|c|}
\hline DNL $(\mathrm{dB})$ & $L_{\mathrm{ct}}=66.3 \mathrm{~dB}(-1 \sigma)$ & $L_{\mathrm{ct}}=73.3 \mathrm{~dB}($ mean $)$ & $L_{\mathrm{ct}}=80.3 \mathrm{~dB}(+1 \sigma)$ \\
\hline 65 & $53 \%$ & $71 \%$ & $86 \%$ \\
\hline 60 & $66 \%$ & $80 \%$ & $94 \%$ \\
\hline 55 & $78 \%$ & $91 \%$ & $98 \%$ \\
\hline 50 & $88 \%$ & $97 \%$ & $100 \%$ \\
\hline 45 & $95 \%$ & $99 \%$ & $100 \%$ \\
\hline
\end{tabular}

The figures seen in Table I will vary somewhat as new empirical information becomes available in the future about the distribution of tolerances for aircraft noise exposure in additional communities. It seems doubtful, however, that the values will vary by more than a few percent. They may therefore serve as approximate guidance for regulatory decisions about threshold values for criteria of "acceptable" noise exposure levels in airport communities.

\section{Conclusions}

Communities with similar noise exposure can differ widely from one another in the prevalence of a consequential degree of transportation noise-induced annoyance. Dosage-response relationships between transportation noise exposure and community annoyance that are derived by correlation (e.g., regression) methods are not the only, nor necessarily the most useful, for transportation planning and regulatory policy purposes. A standardized causal model of annoyance, described in an international technical consensus standard (ISO 1996-1: 2016) that distinguishes between acoustical and non-acoustical influences on self-reported annoyance, accounts for more variance than univariate regression, while furnishing a systematic rationale for regulatory decision making.

\section{REFERENCES}

[1] Federal Interagency Committee on Noise (FICON) Federal Agency Review of Selected Airport Noise Analysis Issues, 
1992. Available online at:

ftp://ftp.agl.faa.gov/Materials\%20Released\%20Related\%20 to $\% 20$ the $\% 20$ OM\%20EIS/3-31-2005\%20World $\% 20$ Gatew ay\%20Related\%20Documents/787 122.pdf

[2] Schultz, T. J. "Synthesis of social surveys on noise annoyance." J. Acoust. Soc. Am. 64, 377-405, 1978.

[3] ISO 1996-1:2016, International Standards Organization, "Acoustics - Description, measurement and assessment of environmental noise - Part 1: Basic quantities and assessment procedures”, Geneva, Switzerland, 2016.

[4] Fidell, S. "A Review of U.S. Aircraft Noise Regulatory Policy," Acoustics Today, 11 (4), 26 - 34, 2015.

[5] Rosenblith, W. A., Stevens, K. N., and the Staff of Bolt, Beranek, and Newman. "Handbook of Acoustic Noise Control. Volume II. Noise and Man." WADC TR-52-204, Wright Air Development Center, Wright-Patterson Air Force Base, Ohio, 1953.

[6] Fidell, S., Mestre, V., Schomer, P., Horonjeff, R., and Reid, T., "A systematic rationale for defining the significance of aircraft noise impacts" J. Acoust. Soc. Am., (2014), 136 (3), 1129-1138. (See also Erratum in J. Acoust. Soc. Am. 140 (4), 2016).

[7] International Standards Organization "Assessment of noise annoyance by means of social and socio-acoustic surveys", ISO Technical Specification 15666, Geneva (2016).

[8] Fidell, S., Mestre, V., Schomer, P., Berry, B., Gjestland, T., Vallet, M., and Reid, T. "A first-principles model for estimating the prevalence of annoyance with aircraft noise exposure", J. Acoust. Soc. Am. 130 (2), 791-806, 2011.

[9] Schomer, P., Mestre, V., Fidell, S., Berry, B., Gjestland, T., Vallet, M., and Reid, "Role of community tolerance level (CTL) in predicting the prevalence of the annoyance of road and rail noise" J. Acoust. Soc. Am., 131(4), 2772-2786, 2012.

[10] Taraldsen, G., Gelderblom, F., and Gjestland, T. "How to measure community tolerance levels for noise" J. Acoust. Soc. Am., 140, 692 (2016).

[11] Schreckenberg, D., and Meis, M. (2007). "Noise annoyance around an international airport planned to be extended," Proceedings of Inter-noise 2007; see also primary German-language technical report "Fluglarm and Gesundheit in der Rhein-Main Region," (Aircraft noise and health in the Rhein-Main area, downloadable from http://www.frankfurt.de/ sixcms/detaffiphp?id=4735799\&_ffmparLid_inhaltj=55556 04 (last viewed April 22, 2011), 1-10.

[12] Vallet, M., Vincent, B., and Olivier, D. "La gêne due au bruit des avions autour des aeroports (The annoyance of aircraft noise around airports), INRETS Report No. LTE 9920, Lyon-Bron, 2000.

[13] Alexandre, A. "Prevision de la gene due au bruit autour des aeroports et perspectives sur les moyens d'y remedier" (Prediction of annoyance due to noise around airports and speculations on the means for controlling it), Anthropol. Appl., Doc. A.A. 28/70, 1-151, 1970. 